Sociologie et sociétés

\title{
Henri Desroche et ses réseaux québécois
}

Entre théorie de l'utopie et pratiques maieuticiennes

Henri Desroche and His Quebec Networks

Between Utopian Theory and Maieutician Practices

\section{Jacques Palard}

Volume 37, numéro 2, automne 2005

Le Québec et l'internationalisation des sciences sociales

Quebec and the Internationalization of the Social Sciences

URI : https://id.erudit.org/iderudit/012911ar

DOI : https://doi.org/10.7202/012911ar

Aller au sommaire du numéro

\section{Éditeur(s)}

Les Presses de l'Université de Montréal

\section{ISSN}

0038-030X (imprimé)

1492-1375 (numérique)

Découvrir la revue

\section{Citer cet article}

Palard, J. (2005). Henri Desroche et ses réseaux québécois : entre théorie de l'utopie et pratiques maïeuticiennes. Sociologie et sociétés, 37(2), 21-47.

https://doi.org/10.7202/012911ar

\section{Résumé de l'article}

L'influence exercée par Henri Desroche sur le processus d'internationalisation des sciences sociales au Québec, du milieu des années 1960 à celui des années 1980, a pris corps de part et d'autre de l'Atlantique. Au Québec même, d'abord, où H. Desroche interviendra principalement à l'École des HEC de Montréal et dans les universités de Sherbrooke et de Rimouski. À Paris également, où de nombreux étudiants québécois vont suivre son enseignement et placer leur recherche sous sa direction au sein du Collège coopératif. L'analyse se fonde sur l'hypothèse selon laquelle la constitution de ses réseaux québécois tient à la relative homologie structurale entre l'individu Desroche, dans son propre parcours personnel et dans ses orientations intellectuelles et praxéologiques, et la culture politique d'un Québec de la fin ou d'après la Révolution tranquille en quête de nouveaux repères en matière d'action collective. 


\section{Henri Desroche et ses réseaux québécois}

Entre théorie de l'utopie et pratiques maïeuticiennes'

\section{JACQUES PALARD}

Centre de recherche et d'étude sur le Canada et

le Québec en sciences sociales

Institut d'études politiques de Bordeaux

Domaine universitaire

33607 Pessac, France

Courriel : j.palard@sciencespobordeaux.fr

$\mathrm{L}$

'ANALYSE RÉTROSPECTIVE DES RÉSEAUX nécessite une forme d'archéologie du savoir et une mobilisation de la mémoire susceptibles d'apporter des réponses à quelques questions clés: quelles ont été les occasions, sans doute en partie contingentes, des initiatives fondatrices et des premiers projets de collaboration? Comment est-on passé du stade de la connivence et de l'affinité à celui de la coalescence puis de la structuration? Un leader s'est-il imposé, ou les relations se sont-elles au contraire établies sur un mode principalement égalitaire? Quelles ont été les productions et les réalisations les plus marquantes du réseau? Surtout, comment rendre compte, en termes de complicité intellectuelle et d'intérêts sociaux partagés, d'une coopération durable?

L'analyse des réseaux universitaires n'échappe pas à un tel questionnement. Lorsque ceux-ci adoptent des formats internationaux, d'autres questions ne manquent d'ailleurs pas d'émerger' ${ }^{2}$. Dans les divers sites nationaux impliqués, est-on ou non en présence d'un développement égal ou similaire des connaissances et des institutions? Que représente intellectuellement et symboliquement l'établissement d'un lien privilégié avec

1. Cette recherche a profité d'une subvention de la Fondation Crédit coopératif (France).

2. Sur la notion de réseau intellectuel, étudiée au travers de l'historicité de milieux franco-québécois et, en l'occurrence, de l'entrelacement des systèmes relationnels d'Édouard Montpetit et d'André Siegfried, voir l'analyse de Gérard Fabre (2004). 
une ou des personnalités étrangères? Par quel processus un paradigme commun peutil se construire? Avec quels types de composantes du système universitaire s'établissent le plus favorablement les relations?...

Certaines de ces questions se rapportent à la nature des acteurs en présence, d'autres aux catégories de temps et de lieu. L'attention est également appelée à se porter sur les inévitables effets de miroir: qu'est-ce que de telles relations nous révèlent des systèmes sociaux et des institutions en présence, et en quoi nous permettent-elles de mieux connaître et comprendre les divers partenaires? C'est ce type d'interrogations complémentaires qui s'est progressivement imposé comme central dans cette étude des réseaux québécois constitués autour d'Henri Desroche, du milieu des années 1960 à celui des années 1980, et qui ont apporté leur contribution, en tant que lieux d'échanges et d'apprentissage, à l'ouverture internationale des sciences sociales au Québec.

L'influence exercée par Henri Desroche de part et d'autre de l'Atlantique s'est d'abord manifestée sur le sol québécois proprement dit, où le sociologue français effectuera, à compter de 1964, de nombreux séjours, sans jamais toutefois s'y installer. Il y interviendra à Montréal, particulièrement à l'École des hautes études commerciales (HEC), sur le thème du mode d'organisation coopérative, mais aussi et surtout dans deux universités «en région»: celle de Sherbrooke et celle de Rimouski. À l'Université de Sherbrooke, il sera appelé à initier puis à conforter la sociologie des religions et, comme à Montréal, celle de la coopération; à l'Université du Québec à Rimouski, il contribuera au lancement d'un programme en développement régional. Cette influence d'Henri Desroche s'est prolongée à Paris, où de nombreux étudiants québécois sont venus suivre son enseignement, le plus souvent pour y placer leur recherche personnelle sous sa direction. Là, son enseignement et ses travaux s'inscrivent dans le cadre du Collège coopératif, qu'il a créé en 1958 (au no 7 de l'avenue Franco-Russe), et dans celui de la VI ${ }^{e}$ section de l'École pratique des hautes études (ÉPHÉ) ${ }^{3}$, devenue, en 1975, l'École des hautes études en sciences sociales (ÉHÉSS).

Pourquoi une influence à la fois longue, diversifiée et efficiente a-t-elle pu ainsi se «traduire» en terre québécoise? L'hypothèse qui s'est peu à peu imposée a trait à la

3. Sur l'influence de la sociologie française au Québec et, plus particulièrement, sur celle de la VI $\mathrm{I}^{\mathrm{e}}$ section de l'École pratique des hautes études, de la fin des années 1950 au début des années 1970, voir Marcel Fournier (1972; 1973, p. 736-748), qui a lui-même préparé et soutenu sa thèse à l'ÉPHÉ sous la direction de Pierre Bourdieu: «Parmi [les] étudiants canadiens [en France], en majorité québécois et de langue française, ce sont principalement des étudiants en sociologie qui s'inscrivent à l'ÉPHÉ: en 1970-1971, la plupart d'entre eux étudient effectivement à cette école (65,6 \%). Il semble donc que l'ÉPHÉ a eu durant les dix dernières années, et a encore, une fonction non négligeable dans la formation de sociologues de langue française: même si dix Québécois y ont obtenu un doctorat de $3^{\mathrm{e}}$ cycle en sociologie, plus de quatre-vingts y ont poursuivi une scolarité de doctorat, sous la direction principalement d'Alain Touraine (18), de G. Friedmann et E. Morin (14), d'Henri Desroche (15), de Lucien Goldmann (12) et de Chombart de Lauwe (7), qui tous sont venus, à une ou plusieurs reprises, enseigner au Québec entre 1960 et 1970. [...] Ces directeurs d'études, qui dirigent les travaux d'étudiants canadiens, sont aussi ceux qui dirigent les travaux du plus grand nombre d'étudiants. De plus, Desroche, Goldmann et Touraine sont ceux dont le nombre d'étudiants d'origine étrangère est plus élevé que celui d'étudiants d'origine française» (Fournier, 1973, p. 744-745). 
relative homologie structurale entre, d'une part, l'individu Desroche, dans son propre parcours personnel - singulièrement religieux — et dans ses orientations intellectuelles et praxéologiques, et, d'autre part, la culture politique d'un Québec qui sort de la Révolution tranquille et qui est en quête de nouveaux repères en matière d'action collective. Ce Québec-là constitue un laboratoire de choix pour l'observateur et une terre de mission pour l'expérimentateur social. Dans un contexte où se mêlent processus de sécularisation et bilan en demi-teinte de la planification régionale engagée par l'État, notamment avec l'expérience fortement discutée du Bureau d'aménagement de l'Est du Québec (BAEQ), Desroche se trouve au diapason et en adéquation avec nombre d'attentes plus ou moins implicites. Il est en effet porteur de clefs d'interprétation en même temps que d'un savoir original par sa transversalité thématique et d'un savoirfaire doté d'une forte capacité de mobilisation. Chercheur de terrain et théoricien, il est dans le même temps formateur, animateur et vulgarisateur, particulièrement attentif aux conditions culturelles - y compris religieuses — du développement économique territorial. À un moment où fleurissent au Québec les analyses en termes de «dépendance», il se démarque, d'ailleurs sans ostentation, en centrant son attention et son énergie sur le devenir des «petites communautés » et des sociétés périphériques, c'està-dire sur des groupes sociaux et territoriaux en recherche tout à la fois d'autonomie et d'outils de développement.

Des institutions universitaires en cours de consolidation vont trouver dans cet homme-orchestre l'instigateur et le vecteur d'une spécialisation disciplinaire et d'un ancrage à la fois mieux affirmé et durable dans leur environnement régional. Cette perspective conduit à inscrire les réseaux québécois d'Henri Desroche dans une approche inspirée de l' «interactionnisme structural», approche spécifique à l'analyse de réseaux qui entend analyser les relations d'influence réciproque entre l'individu et les structures sociales dans lesquelles il se trouve inséré (Degenne et Forsé, 1994, p. 13-16). Avec Vincent Lemieux, on considérera les réseaux comme des «systèmes d'acteurs sociaux qui, pour des fins de mise en commun de la variété dans l'environnement interne, propagent la transmission de ressources en des structures fortement connexes» $\left(1999\right.$, p. 11) ${ }^{4}$. Ainsi que le souligne Marcel Fournier, la rencontre entre le maître et ses disciples ne saurait être le fait du hasard:

La transmission des informations [scientifiques] est aussi fonction de leurs conditions sociales de production, de transmission et de réception, de telle sorte que l'on peut comprendre les phénomènes de diffusion, d'emprunt ou d'échange international [...] seulement à condition de subordonner l'analyse de la logique des interactions entre chercheurs ou groupes de chercheurs à la construction des relations objectives entre les positions qu'ils occupent dans le champ scientifique et donc à l'étude des fonctions que remplissent ces diffusions, emprunts ou échanges pour les chercheurs ou catégories de chercheurs en situation de concurrence pour la légitimité culturelle. (1973, p. 700-701)

4. Vincent Lemieux définit la notion de réseaux par rapport à celle d'appareils, ceux-ci étant considérés comme «des systèmes d'acteurs sociaux qui, pour des fins de mise en ordre de la variété dans leur environnement externe, contraignent la transmission de ressources en des structures faiblement connexes» (p. 11). 
Deux dimensions complémentaires permettent d'appréhender la vigueur et l'originalité des réseaux québécois d'Henri Desroche: une dimension sociohistorique, qui permet de comprendre sur quelles valeurs et sur la base de quelles conceptions partagées a pu s'engager son intervention dans le contexte de la société québécoise de l'aprèsRévolution tranquille; une dimension scientifico-universitaire, qui se matérialise par l'émergence de nouveaux thèmes ou terrains de recherche, en particulier dans le cadre de la préparation de thèses de doctorat sur la religion, le coopérativisme et le développement régional. Le traitement de ces thèmes se traduit par une démarche de nature méthodologique d'élucidation et de théorisation de la pratique et, conjointement, par une forme d'engagement du chercheur sur le terrain de l'action, à la fois localerégionale et internationale.

Cet effort d'élucidation ne doit pas cacher la difficulté de l'entreprise, qui tient à la personnalité pour le moins riche et complexe d'Henri Desroche. Le sous-titre de l'ouvrage d'hommages qui lui a été consacré en 1997, trois ans après sa mort, dit bien son caractère profondément atypique: «un passeur de frontières». Les frontières que franchit Henri Desroche sont bien sûr géographiques, autant continentales que nationales; mais elles sont aussi thématiques, disciplinaires et, surtout peut-être, professionnelles tant a importé à ses yeux la subversion de la ligne de partage qui sépare ceux qui sont censés savoir de ceux qui sont censés apprendre. En tout cela, Henri Desroche se veut un itinérant et un mutant. Sociologue à la fois, on l'a vu, de la religion, des coopératives et du développement régional, thèmes auxquels il convient d'adjoindre celui de l'éducation permanente, il a eu dans l'ensemble de ces domaines, dont aucun n'a échappé à ses incursions québécoises, une activité éditoriale des plus fécondes: on compte au total une cinquantaine d'ouvrages et pas moins d'un millier d'articles ${ }^{5}$. Le moins que l'on puisse dire c'est qu'Henri Desroche est inclassable, si l'on s'en tient aux critères communément admis du cursus universitaire: il pratique avec délectation, moins sans doute par choix délibéré que par tempérament, un mélange des genres qui n'est pas, loin s'en faut, sans effet sur l'influence voire la fascination qu'il a exercées et dont on l'a, à juste titre, généreusement crédité. À cela s'ajoute un art de la communication écrite et orale qui aime à cultiver sinon l'hermétisme du moins la sophistication ${ }^{6}$.

5. Henri Desroche situe ses recherches à la confluence d'une double orientation: d'une part, des «recherches culturelles sur l'imagination constituante, à travers messianismes, utopies, prophétismes, millénarismes, idéations collectives, effervescences culturelles»; d'autre part, « des recherches socioéconomiques sur la pratique instituée par les associations coopératives, communautaires, mutuellistes, syndicales ou parasyndicales» (1978a, p. 7-8).

6. Deux exemples, pris parmi beaucoup d'autres, permettent d'illustrer les fioritures de son style. Le premier est emprunté à la lettre qu'il adresse aux organisateurs du colloque Les villages ruraux menacés: le pari du développement, organisé en septembre 1988 à la Trinité-des-Monts (comté de Rimouski) par le Groupe de recherche interdisciplinaire en développement de l'Est du Québec (Université du Québec à Rimouski) et l'Institut de développement Nord-Sud (animé par Violette Gendron à La Pocatière) dans le cadre de l'Université coopérative internationale; Henri Desroche y évoque l'automne québécois et y parle des scènes et des scénarios dont sa mémoire «est affectivement affectée et qui, pour elle, demeurent campés dans le décor [des] liturgies forestières lorsque, mutantes à l'approche des frimas, elles se mettent à processionner selon leur cérémonieux métissage entre les pérennités de leurs verdoyances et les éphémères somptuosités de leurs pourpres» (GRIDEQ, 1989, p. 215). Le second échantillon est tiré de l'analyse qu'il effectue du pre- 


\section{1- LES CONDITIONS SOCIOHISTORIQUES DE LA CONSTITUTION DES RÉSEAUX}

«Avoir des amis, écrit Hobbes dans le Léviathan, c’est avoir du pouvoir.» On pourrait avancer que bâtir ou rejoindre un réseau, c'est plutôt choisir de partager le pouvoir et ses savoir-faire dans le cadre d'une action collective qui prend la forme d'un jeu coopératif, sans toutefois que soient par avance déterminées les modalités de la répartition des gains éventuels.

Deux de mes interlocuteurs québécois ${ }^{7}$, anciens disciples ou très proches collaborateurs d'Henri Desroche, aujourd'hui encore en poste de responsabilité dans des universités québécoises, ont l'un et l'autre cru devoir conclure sur le mode d'un quasi-démenti un entretien d'environ trois heures, à la fois documenté et passionné et qui venait précisément, me semblait-il, de démontrer tout le contraire: «Henri Desroche n'a pratiquement rien laissé, du moins rien de tangible/rien de palpable»! Ils entendaient signifier par là que l'action d'Henri Desroche s'est révélée plus édifiante celle d'un maître à penser - qu'édificatrice. De fait, il n'a rien coulé dans le béton et ne s'est que fort peu intéressé à l'institutionnalisation de ses innovations; mais, malgré cela ou, plutôt, pour cette raison même, il a profondément marqué les esprits qui l'ont durablement fréquenté. S'il fallait le situer dans un triptyque biblique qu'il n'aurait sans doute pas désavoué: prêtre, prophète et roi, c'est à coup sûr du côté de la prophétie que l'auraient porté à se définir à la fois le contenu de son enseignement, ses pratiques didactiques et sa conception des échanges et des liens réticulaires.

Parler de «réseaux» à propos d'Henri Desroche, c'est évoquer à la fois le mot et la chose. Il convient en effet de souligner que ce terme fait partie non seulement de son langage courant mais aussi de sa façon habituelle et délibérée de procéder: il insiste volontiers sur la circulation du savoir, la plasticité des relations et le partage des expériences. Il aura passé sa vie à tisser des liens et à faire tomber les frontières. Henri Desroche n'est pas un bâtisseur; c'est un animateur et, plus encore, un «maïeuticien», un «accoucheur», un «sage-homme», pourrait-on dire. Parler de réseaux à son endroit, c'est dire qu'à ses yeux le processus l'emporte toujours en importance sur le résultat: Desroche est assurément un «processeur» avant que d'être un professeur. Sa façon de définir, à la fin de sa vie, son propre parcours, dans sa revue Anamnèses, a beaucoup à voir avec cette conception et cette interprétation de sa propre posture:

mier «septennat» de l'Université coopérative internationale; évoquant tour à tour le projet forgé par Charles Gide dans les années 1930, l'inaboutissement de l'International Council for Research in CooperationDevelopment et la création, en 1958, du Collège coopératif, il évoque une «triple prégnance»: «une prégnance archétype après coup; une prégnance heuristique obsolète; une prégnance collégiale permanente» (Desroche, 1984, p. 11).

7. Les chercheurs qui ont accordé un entretien à l'auteur sont: Claude Beauchamp, Université Laval; Bernard Denault, Université de Sherbrooke; Hughes Dionne, Université du Québec à Rimouski; Gabriel Dussault, Université Laval; Alain G. Gagnon, Université du Québec à Montréal; Violette Gendron, Institut de développement Nord-Sud; Fernand Harvey, INRS-Urbanisation, Culture et Société; Juan-Luis Klein, Université du Québec à Montréal; Danielle Lafontaine, Université du Québec à Rimouski; Benoît Lévesque, Université du Québec à Montréal; Paul Prévost, Université de Sherbrooke; Marc-Urbain Proulx, Université du Québec à Chicoutimi; Benoît Tremblay, École des HEC de Montréal; Lucien Vachon, Université de Sherbrooke. 
Qui parle et d'où parle-t-il ? [...] 1 - Il s'agit d'un vieux «prof» [...] et il parle à partir d'un établissement d'enseignement supérieur, l'ÉHÉSS; nonobstant 10 années préalables, il y a vieilli sous le harnais de trente années en école semi-buissonnière, à minimum de scolarités, en optimum de créativités, moyennant une pédagogie contractuelle, bienheureusement délestée de lourdeurs docimologiques dans les jeux et les enjeux de ses partenariats. 2 - À cette position de «directeur d'études» pendant trois décennies, s'adjoint, même durée, un positionnement collégial et associatif: celui d'un Collège coopératif-Paris [...]. 3 - Cette innovation collégiale s'est redéployée dans un réseau coextensif à l'Hexagone [...]. 4 - De ce réseau en hexagone à un réseau sur mappemonde: en l'occurrence une Université coopérative internationale [...]. 6 - Au bout du compte, une cohorte d'auteurs-acteurs: auteurs de recherches sur les actions dont ils ont été, sont ou seront les acteurs... et réciproquement. Un grand millier de leurs prestations dans mon conservatoire. Et autant de parcours qui se sont accomplis non pas en consommant des cours mais en produisant des savoirs.» (Desroche, 1990, 38)

L'importance qu'il accordera au concept d'université «extra-muros» dit bien qu'il se donne à voir comme un fondateur à l'évidence peu soucieux de construction administrative.

Dans l'ouverture à l'ouvrage d'hommages qui lui est dédié, Émile Poulat évoque le goût pour le compagnonnage de ce maître original et inclassable, «qui n'enseigne pas à un public, mais qui éveille à lui-même chacun de ceux qui l'écoutent, et qui, au lieu de s'enfermer dans sa salle de cours, va sur place, explore le terrain, pérégrine à travers le monde, et fonde sans relâche» (Poulat, 1997, p. 10). Quelques pays ou régions du monde auront sa préférence: l'Afrique, l'Amérique latine, certains pays d'Europe orientale, Israël et le Québec.

\section{Le développement des sciences sociales au Québec}

Parmi les évolutions qui caractérisent la Révolution tranquille, deux sont mises en exergue par Gilbert Renaud qui permettent de comprendre le contexte dans lequel s'inscrivent les interventions de Desroche. D'une part, on assiste à la poursuite de l'autonomisation du champ scientifique par rapport au discours religieux, particulièrement dans le domaine des sciences sociales; ainsi, se structure « un nouveau modèle culturel qui fait passer du progrès par l'industrialisation au développement par l'acquisition et l'application de la connaissance scientifique» (Renaud, 1984, p. 65). En outre, se développe une société de participation, qui profite de la croissance économique et qui contribue à ce que s'amorce « un nouveau type d'intervention auprès des classes populaires des quartiers urbains et des régions éloignées. De partout naissent des comités de citoyens où l'on retrouve un personnage central: l'animateur social. [...] Désormais, la connaissance sera mise au service du peuple et surtout de ses fractions les plus pauvres qui sont invitées à faire valoir leurs droits et à s'intégrer dans la nouvelle société en construction » (p. 71). Au travers de la contestation à la fois de l'orientation et de la gestion du développement, c'est l'entrée des couches populaires dans la nouvelle société en construction qui se joue. 
Au cours des années 1970, le débat sur la nature de la société québécoise et, particulièrement, sur son degré de dépendance et sur les conditions structurelles du développement scientifique revêt une importance cruciale. Pour Louis Maheu, «le Québec n'est pas une société libérale dominante générant, contrôlant, gérant son propre développement économique» (Maheu, 1980, p. 20). Société développée, le Québec n’en constituerait pas moins ainsi une société dépendante; Alain Touraine estime d'ailleurs que son niveau de modernisation «ne constitue d'aucune manière un obstacle à l'application au Québec des principes d'analyse valables pour l'ensemble des sociétés dépendantes», l'essentiel étant alors de rappeler que «les trois composantes des luttes historiques: classe, nation et modernisation se croisent de manière encore plus complexe dans un pays hautement développé comme le Québec que dans les autres sociétés dépendantes» (Touraine, 1978, p. 182-183).

Dans le déploiement de cette réflexion, deux contributions permettent de mieux saisir la situation structurelle et le mode de production des sciences sociales au Québec et ainsi de comprendre la place que la pensée et les modes d'intervention d'Henri Desroche pourront y occuper. La première se situe dans le cadre du colloque organisé au cours de l'été 1976 sur le campus de l'Université Bishop de Lennoxville autour de l'œuvre sociologique d'Alain Touraine. Au cours de cette rencontre, s'engage un affrontement sur les défis posés à la sociologie des rapports sociaux, en particulier sur la façon dont la pratique sociologique peut rendre compte de conduites de contestation et de rupture sociales ${ }^{8}$. Jacques Dofny défend ainsi l'idée d'une double dépendance du Québec: la Province dépend du Canada, qui dépend lui-même des États-Unis (Dofny, 1978, p. 88). Se fondant sur un tel statut de dépendance du Québec, tant dans l'économie nord-américaine que dans le champ scientifique international, Gabriel Gagnon estime que «les sciences sociales au Québec, particulièrement la sociologie à laquelle ses méthodes permettent moins pour le moment de s'abstraire des projets des acteurs, loin de constituer un univers à part défini exclusivement par des critères de validité interne, sont éminemment liées à l'univers des mouvements sociaux» (Gagnon, 1978, p. 116).

La seconde contribution tient en une explicitation de la position et des fonctions des sciences sociales au Québec. Marcel Fournier et Louis Maheu observent au milieu des années 1970 que le développement et l'institutionnalisation de ces disciplines sont enracinés dans l'évolution de la société québécoise des décennies précédentes: la transformation des rapports entre, d'une part, le champ politique et le champ scientifique et, d'autre part, le champ politique et d'autres champs tels que les champs religieux et intellectuel conditionne le développement des sciences sociales: «De telles incitations structurelles demeurent reliées à l'ensemble des positions sociales occupées par les spécialistes de ces disciplines au moment où ce sous-champ scientifique ne peut, au nom de sa logique propre de fonctionnement, exclure dans la définition des rapports qu'il

8. Les actes ont été publiés dans Sociologie et sociétés, vol. 10, $\mathrm{n}^{\circ} 2,1998$, numéro intitulé: Changement social et rapports de classe. 
entretient avec d'autres champs les prescriptions relatives aux positions politiques et administratives tenues par divers agents scientifiques» (Fournier et Maheu, 1975, p. 107) $)^{9}$.

C'est dans ce contexte qu'il convient de situer les liens qui s'instaurent entre le Québec des années 1960 et 1970 et les pays où vont se former ses spécialistes universitaires en sciences sociales. Dans ce dispositif, Paris occupe une place relativement dominante: ainsi, sur 28 anciens étudiants du Département de sociologie de l'Université de Montréal qui ont préparé et soutenu une thèse de doctorat entre 1962 et 1975, 14 l'ont fait dans une université parisienne ou à l'ÉPHÉ ${ }^{10}$.

\section{Henri Desroche et la rémanence de l'utopie dans une société sécularisée}

Pour Alain Touraine, «Henri Desroche est la figure centrale d'un vaste mouvement culturel qu'on pourrait nommer la sécularisation de l'eschatologie religieuse » (Touraine, 1997, p. 17). Au centre de ce mouvement, se situe l'idée de communauté, qui se réfere moins à la défense d'un héritage culturel qu'à un idéal d'autogestion du changement. Le parcours individuel d'Henri Desroche, qui quitte l'ordre dominicain à la suite de la publication en 1949 de son ouvrage Signification du marxisme et qui s'engage, à l'École pratique des hautes études, dans une recherche sur les Shakers américains (Desroche, 1955), est pour une part l'incarnation de ce processus de sécularisation. L'histoire religieuse du Québec est alors elle-même marquée par l'affaiblissement rapide des pratiques religieuses prescrites et le départ de nombreux prêtres, religieux et religieuses des fonctions ecclésiastiques. Ce processus de sécularisation et de décléricalisation prend la forme corrélative de la modernisation de l'État. Le Québec qu'il est donné à Henri Desroche de rencontrer et d'étudier vit ainsi une expérience de transformation nationale originale, que son propre itinéraire lui permet de comprendre de l'intérieur. C'est dire que sa façon d'y travailler et d'y intervenir est riche d'enseignements sur le Québec même, à un moment où s'y développent de nouvelles institutions publiques et, plus particulièrement, un réseau scolaire et universitaire. Le Québec connaît en effet une période «sensible» où ce mouvement de réforme prend appui sur la plasticité des esprits et l'aptitude au changement. Le temps est propice à l'utopie et au rêve et, plus largement, à la quête de nouveaux repères. Mais, dans ce mouvement de déprise de l'institution et du magistère catholiques, la matrice religieuse de la société québécoise ne disparaît pas pour autant; elle se transforme plutôt en des substituts fonctionnels dont la fonction première sera de continuer à faire vivre l'idée de «communauté», que celle-ci s'incarne dans la nation ou dans l'action locale. L'un des tout premiers écrits d'Henri Desroche, quelques années avant de quitter, en 1951, l’ordre des

9. Voir également Fournier, Germain, Lamarche et Maheu (1975).

10. Sociologie et sociétés, vol. 7, $\mathrm{n}^{\circ} 2$, nov. 1975, p. 151-152. C’est le cas précisément des thèses de doctorat, soutenues à l'ÉPHÉ, de Marcel Fournier, Institutionnalisation et différenciation de disciplines dans une situation de double dépendance (1973), sous la direction de Pierre Bourdieu, et de Louis Maheu, Enseignement supérieur et structure sociale: les fonctions sociales de l'Université québécoise (1974), sous la direction d'Alain Touraine. 
Frères prêcheurs, alors qu'il collabore à l'équipe d'Économie et humanisme, porte sur la «dialectique de la communauté»:

Avant toute autre chose, écrit-il, et dans la nudité de son expression philosophique, le problème de la communauté est un problème de la réduction du multiple à l'un. Il est ce problème-là, envisagé dans le cas humain. À la limite [...], la Communauté n'est autre chose que la réduction parfaite à l'Unité d'une ou de la Multiplicité humaine. [...] L'idée communautaire apparaît comme la formule de l'immense retour à Dieu de toutes choses. Elle dort dans les agrégats inertes, rêve dans les sociétés animales, s'éveille dans les innombrables manières préconisées par les hommes pour vivre ensemble. (Desroches, 1944 , p. 5 et $22^{11}$ )

Le succès de l'expression «action communautaire» trouve dans ce système de représentations de l'organisation sociale, qui associe intimité et universalité, l'un de ses fondements et de ses ressorts les mieux assurés. Par communauté, on peut en effet entendre une organisation de production ou de services animée par un principe d'autogestion et d'égalité et qui porte en elle la même dualité que la sécularisation de l'eschatologie: le recours à un principe métasocial et l'entrée dans le monde de l'action historique réelle (Touraine, 1997, p. 18). La sacralisation du social peut ici constituer le corollaire de la sécularisation de l'eschatologie. En ce sens, l'utopie cherche à s'incarner dans un modèle social qui permet d'associer, à l'instar de l'idéal coopératif, activité économique et protestation sociale et morale, gestion et contestation. On perçoit ici la volonté de réinvestir le patrimoine socioéconomique et culturel dans un processus de modernisation en contexte collectif et démocratique: pas de projet sans héritage, pas d'espérance sans mémoire. À l'instar d'une religion, le projet s'inscrit d'une certaine façon dans une lignée croyante et la sociologie peut vouloir se mettre au service d'une telle perspective. Ainsi, selon le parallèle qu'établit Jean-Jacques Simard, «alors que la religion aura été, selon le mot de Durkheim, "la science des sociétés sans science", les sciences sociales voudront d'abord se constituer en religions des sociétés sans religion » (Simard, 1979, p. 7). Dans cette entreprise, l'utopie est centrale; Henri Desroche, inspirateur de la création, au couvent dominicain de La Tourette, dans la région lyonnaise, du Centre Thomas-More (du nom, symboliquement choisi, de l'auteur d'Utopie $)^{12}$, se montrera parfaitement en prise avec une telle prédisposition qu'il qualifie d' «imaginactive». Sur la thématique de la substitution des sciences sociales aux traditions, de l'opposition entre l'homme «naturel» et l'homme «savant», de l'extension des pouvoirs technocratiques par l'utilisation des sciences humaines et de la normativité de la science - au travers notamment du taylorisme —, il engage un débat avec Fernand Dumont; cet échange donnera lieu à un enregistrement réalisé à Montréal

11. À la même période, Henri Desroche s’intéresse à la communauté de travail Boimondau, sur laquelle il entreprend une recherche qu'il reprendra quelques années plus tard avec Albert Meister. Selon une vision à tendance totalisante, la communauté de travail est définie à la fois comme mutualité, coopérative et syndicat (Desroche et Meister, 1955).

12. Henri Tincq, «Le béton de la passion», Le Devoir, 6 janvier 1996. 
en juin 1975 et diffusé en septembre suivant sur les ondes radiophoniques de FranceCulture ${ }^{13}$.

Lorsqu'en 1973 Henri Desroche publie sa Sociologie de l'espérance, il se trouve pleinement engagé dans ses réseaux québécois. Le mode d'inscription qu'il y adopte, l'enseignement qu'il y délivre tout comme ses interventions sur le terrain ne peuvent pas ne pas se ressentir de sa conception du messianisme et de l'utopie qu'il rend ainsi publique. Pour lui, les relations qui s'instaurent entre le message (religieux) et le programme (politique) devraient être placées en perspectives réciproques: en effet, si le message est en un sens l'involution du programme, le programme ne pourrait pas être lui-même autre chose que la retombée sociologique du message: «Le fait messianique se trouve généralement moins devant un dilemme (réussir ou échouer) que devant un destin (réussir et échouer). Sa dialectique n'est pas circulaire, comme si son essence lui demandait de boucler sur lui-même; elle serait plutôt spirale, cette essence impliquant congénitalement l'échec représenté par le fait qu'elle s'échappe à elle-même. [...] Il n'y a peut-être dans l'histoire que des messianismes ratés» (Desroche, 1973, p. 148). Mais, pour Desroche, un messianisme raté n'en demeure pas moins un messianisme, nécessairement produit par la force d'attraction et de dynamisation exercée par des idées, qu'une raison raisonnante pourrait tenir trop facilement pour factices, fantaisistes, voire pernicieuses, et par le pouvoir dont se saisit l'imagination, dont le tremplin est souvent de nature religieuse (p. 231).

\section{2- CONSTRUCTION SOCIOLOGIQUE DE LA RÉALITÉ QUÉBÉCOISE ET FORMATION PAR}

\section{LA RECHERCHE}

La diversité thématique de ses terrains de recherche fait d'Henri Desroche un enseignant et un chercheur protéiforme quelque peu atypique; elle lui permet d'enrichir son rôle de formateur et d'animateur par la pluridisciplinarité dont il est porteur. On l'a vu, ses travaux s'inscrivent dans trois domaines: religion, coopératives et développement régional, et chacun de ceux-ci profite de sa fonction d'animateur — certains diraient d'«agitateur» — intellectuel.

\section{La religion comme champ disciplinaire}

Henri Desroche excelle dans l'approche interdisciplinaire de la religion. Son approche du champ religieux le conduit à incorporer ses objets d'analyse dans leur environnement et à les considérer comme des productions sociales. En bref, on pourrait avancer qu'il adopte une approche fonctionnelle et non substantive des phénomènes religieux, et qu'il s'impose un solide travail d'objectivation. On trouve une bonne illustration de ce mode de problématisation dans sa recherche de doctorat sur le messianisme shaker. Chacune des quatre séquences qu’il dégage — Royaume, Église, Ordre et Secte — peut

13. En 1965, à l'occasion de son enseignement à l'École pratique des hautes études, à Paris, F. Dumont est invité à participer aux séminaires d'Henri Desroche (Dumont, 1997, p. 149). 
être rapportée au contexte de son émergence et de son développement, même si elle est «lue» par les acteurs religieux en termes de significations religieuses (sur un mode mystique, kérygmatique, apologétique ou commémoratif), selon une théologie spécifique des réalités terrestres.

Chacun de ces temps, écrit-il, peut être daté, circonstancié, connoté par ses corrélations avec des phénomènes sociaux, économiques ou politiques aussi divers que la condition prolétarienne dans la banlieue de Manchester, les mouvements migratoires aux États-Unis, la guerre américaine d'Indépendance, la poussée vers l'ouest et ses colonisations agricoles, l'industrialisation commençante et ses premiers mouvements sociaux, la guerre de Sécession et ses conséquences économiques, l'industrialisation accélérée et la rupture des économies d'autarcie. (Desroche, 1973, p. 131)

Lorsqu'il écrit dans sa célèbre Sociologie de l'espérance (1973) qu'à la base d'une revendication d'émancipation on doit remarquer une double frustration: frustration économique et sociale et frustration religieuse, on peut penser que son regard le porte alors vers le Québec: «Cette dimension, écrit-il en effet, s’aperçoit dans le fait même que l'oppression ou la dénomination et l'occupation étrangères viennent jouer un rôle fondamental à travers les questions de langues, de lignées, de patrie, d'indépendance ou de dignité nationales, bref, à travers toutes conditions impliquant aliénation ou désaliénation proprement culturelles» (Desroche, 1973, p. 146). Henri Desroche énoncera souvent l'idée selon laquelle l'homme religieux construit les dieux qui le construisent (Desroche, 1973, p. 230).

L'enseignement qu'il délivrera en sciences sociales des religions s'inscrit dans un Québec où l'Église catholique, on l'a vu, perd rapidement de son emprise sur l'organisation sociale. Le besoin s'exprime alors d'une formation universitaire qui puisse échapper au contrôle institutionnel de l'orthodoxie. Lors du colloque interuniversitaire et interdisciplinaire de Sciences religieuses qui se tient à Québec en avril 1968, est ainsi soulignée «la distinction à introduire, le plus rapidement possible, entre la théologie et les sciences de la religion. [...] Il y aurait, en effet, de nombreux avantages à fonder un Institut des Sciences de la Religion dans le cadre d'une Faculté des Sciences humaines» (Ryan, 1969, p. 110) ${ }^{14}$. Il est alors fait état par deux représentants de l'Université de Sherbrooke d'un projet d'intégration d'un premier enseignement en sciences humaines des religions dans le programme d'étude de leur faculté de théologie. Ce projet a en fait connu, dès la rentrée universitaire de 1966, un début de réalisation; il a été conçu et mis en œuvre à la suite d'une rencontre entre Lucien Vachon, doyen depuis mars 1965 de la toute nouvelle faculté canonique de théologie ${ }^{15}$, et Henri Desroche. Ceux qui relatent cette innovation, trente ans plus tard, gardent en mémoire les conditions sociohistoriques et les enjeux universitaires et intellectuels de sa mise

14. Sur la contribution des membres de la faculté des sciences sociales de l'Université Laval, à commencer par son doyen, le R. P. Lévesque, à la dissociation du «social» et du «religieux», voir l'analyse de Marcel Fournier (1973, p. 225-285).

15. Lucien Vachon demeurera doyen jusqu'en 1977; il le redeviendra de 1986 à 1994. 
en œuvre (Dion et Mélançon, 1996). Le contexte est original: à un moment où la clientèle étudiante est exclusivement cléricale, on demande à Lucien Vachon de fonder une faculté universitaire de théologie alors même que les évêques maintiennent le monopole des grands séminaires. De ce fait, les premiers étudiants, en septembre 1966, sont tous laïques, situation qui prévaut jusqu'à ce que, à compter de 1969, les séminaristes soient autorisés à fréquenter l'université.

Là s'enracine le parti pris universitaire de l'enseignement théologique facultaire: la théologie a rang de science, et, dans le même temps, l'accent est mis sur sa fonction sociale et culturelle, à un moment, celui de la Révolution tranquille, où il importe de participer aux grands courants de pensée et d'impulser une conception globale de la réalité sociale. Deux domaines permettent d'innover: le développement de la formation en pastorale scolaire, fondée sur une pédagogie qui prend en compte les «besoins du milieu », et l'introduction des «sciences humaines de la religion». Sur ce point, dès 1968 et jusqu'en 1973, la faculté offre, avec la collaboration du Groupe de sociologie des religions de Paris, qui assure une grande partie des cours, un programme de maîtrise en sciences humaines des religions:

Henri Desroche, Jean Séguy, Jean-Pierre Deconchy, Jacques Maître, Roger Bastide sont invités à participer à cette expérience unique, certainement avant-gardiste, de la confrontation entre théologie et sciences humaines des religions. Pour Lucien Vachon, cette décision voulait produire un "décapage" de la théologie, l’obliger à être vraie et libre, sans repli sur elle-même. [...] Une telle expérience, un peu risquée, évoluera non sans chocs et difficultés, mais, en plus de contribuer à une formation rigoureuse et moderne des étudiantes et étudiants, elle permettra à la théologie de s'affirmer. (Dion et Mélançon, p. 31-32)

Les objectifs sont clairs: développement de la fonction critique du savoir, recherche libre de la vérité théologique et religieuse, inscription dans le mouvement global de la culture universitaire, enrichissement de la théologie grâce à l'apport des sciences qui abordent la religion sous d'autres angles, conception plurielle et à visée synthétique des approches de la vérité. On perçoit là l'enjeu du débat et de la confrontation entre sciences sociales et sciences religieuses, enjeu particulièrement aigu au Québec dans la mesure où, selon Jean-Pierre Deconchy et Gilles Martel, «c'est [...] là seulement, que la confrontation, chez les mêmes individus et dans les mêmes groupes sociaux, du discours théologique et du regard que les sciences humaines portent sur ces discours et leurs objets a été institutionnalisée» (Deconchy et Martel, 1974, p. 6-7). Ce constat fonde la question autour de laquelle s'organise l'investigation sociopsychologique des effets de cette confrontation entre deux types de procédures cognitives: «Comment un groupe religieusement acculturé fonctionne-t-il lorsque, par suite de la nouvelle conjoncture scientifique, il doit s'affronter à une recherche qui veut expliquer sa propre activité religieuse et ses objets sacrés comme produits par l'homme selon des déterminismes naturels?» (p. 4).

La conception qu'impulsent Henri Desroche et ses collaborateurs français du Groupe de sociologie des religions - laboratoire qu'il a contribué à créer une dizaine 
d'années plus tôt et qui relève du Centre national de la recherche scientifique — induit une professionnalisation des sociologues de la religion et l'acceptation de la fonction critique des sciences de la religion ${ }^{16}$. Les thèses de doctorants québécois qu'il dirige en ce domaine, et qui traitent principalement de messianismes de nature religieuse, participent de cette approche. On en a une juste illustration avec les recherches de Gabriel Dussault sur le curé Labelle (1975), de Gilles Martel sur Louis Riel (1976) et de Benoît Lévesque sur les Clercs de Saint-Viateur (1975). Sa référence explicite à Henri Desroche conduit Gabriel Dussault à étudier «l'attestation d'une société éclatante dans la contestation d'une société éclatée»; il emprunte à Jean Séguy sa définition de l'utopie, dont il souligne la parenté avec le messianisme: «Tout système idéologique total visant, implicitement ou explicitement, par l'appel à l'imaginaire seul (utopie écrite) ou par passage à la pratique (utopie pratiquée), à transformer radicalement les systèmes sociaux globaux existants» (Séguy, 1971, p. 331). En introduction à son étude du messianisme que porte le groupe métis de la Rivière-Rouge, Gilles Martel pose l'hypothèse selon laquelle «toute société s'efforce, tant bien que mal, d'équiper ses membres d'un système de réaction qui puisse non seulement amortir [les] chocs frustratoires mais aussi produire de l'énergie de relèvement; c'est un prérequis fonctionnel à la survie de tout groupe humain» (Martel, 1976, p. 10). Le rapport à l'utopie est également au centre de la thèse de Benoît Lévesque, qui organise sa recherche doctorale — « contribution positive à une sociologie génétique des ordres religieux» (p. XIV) — autour d'une double thématique: la genèse d'une congrégation religieuse comme groupement marginal, protestataire et utopique, et sa transformation en groupement intégré à l'Église et à la société, stade de routinisation du charisme. La double direction de fait assurée par Henri Desroche et Jean Séguy, engagés alors l'un et l'autre dans des travaux sur l'utopie et les «sociétés imaginaires», rend compte du choix de sa problématique $^{17}$ : comme Jean Séguy, Benoît Lévesque estime que «l'ordre religieux, à l'égal de la secte, représente une des tentatives que fait une société affectée par le changement pour se restructurer» (Séguy, 1971, p. 340) $)^{18}$.

16. S’inspirant de la comparaison développée par Paul Claudel entre animus et anima (Claudel, 1965), entre l'esprit scientifique et l'expérience religieuse, et répondant au théologien Moltmann qui se demande si la sociologie n'est pas une grande entreprise messianique, Henri Desroche avance que la fonction la plus évidente des «sciences de l'homo religiosus» est leur fonction négative: elles apprennent «pour le moins à une expérience religieuse à ne pas se donner pour une explication scientifique donc à ne pas se substituer à elle, à ne pas prétendre la tenir en tutelle et, à la limite, à ne pas l'évacuer ou la réprimer» (Desroche, 1972, p. 218).

17. Benoît Lévesque parle, à propos de son engagement en thèse de doctorat, d'un heureux concours de circonstances: «Ces circonstances n'étaient autres que les séjours en sol canadien d'Henri Desroche et de Jean Séguy comme professeurs invités au Département des sciences humaines des religions de l'Université de Sherbrooke» (Lévesque, 1975, p. XIX).

18. Les communautés religieuses sont au centre d'autres travaux conduits en parallèle, également dans la mouvance d'Henri Desroche: Gabriel Dussault et Gilles Martel (1976) consacrent ainsi une recherche à la définition et à la mesure du rôle de l'économie dans le développement et la transformation des communautés religieuses au XIX ${ }^{e}$ siècle; dans une perspective similaire, Bernard Denault et Benoît Lévesque (1975), qui travaillent dans le cadre d'un programme «religion et développement au Québec», posent une double hypothèse: la première concerne la fonction d'intégration sociale des fondations féminines québécoises au XIX ${ }^{e}$ siècle; la seconde a trait à la fonction protestaire/attestataire des communautés religieuses; l'intérêt se porte également sur l'émigration — utopique? — des communautés religieuses françaises au Québec au XIX siècle. 


\section{Les coopératives comme modèle des rapports sociaux}

L'institution sociale que constitue la coopérative n'est pas seulement productrice de biens ou de services, elle est aussi porteuse de sens puisque sa spécificité tient idéalement au partage du pouvoir et à celui de la valeur ajoutée. Elle participe des projets imaginaires ou utopiques de construction d'une société alternative ${ }^{19}$.

C'est dans ce domaine de la recherche et de l'enseignement sur le système coopératif qu'Henri Desroche effectuera ses premières interventions au Québec. Il inaugure en effet la chaire de coopération du Département de science économique, alors dirigé par le jésuite Émile Bouvier, que l'Université de Sherbrooke crée en 1967 à la suite d'une entente avec le Conseil de la coopération du Québec. Le directeur de la chaire, Claude Pichette, entend en effet collaborer avec des disciplines sœurs; d'où l'invitation du sociologue Henri Desroche, qui assure un cours de trois semaines durant l'automne 1967 et qui renouvellera l'expérience au printemps suivant lors de la session intercoopérative organisée par l'Institut coopératif Desjardins. Ces deux enseignements ont été rassemblés dans une même publication, qui forme le premier des Cahiers de la coopération publiés par la chaire (Desroche, 1969).

Dans le cadre de ces formations, Henri Desroche s'attache en particulier à promouvoir son modèle du «quadrilatère coopératif», qui a généré de multiples débats et laissé de solides souvenirs dans les esprits:
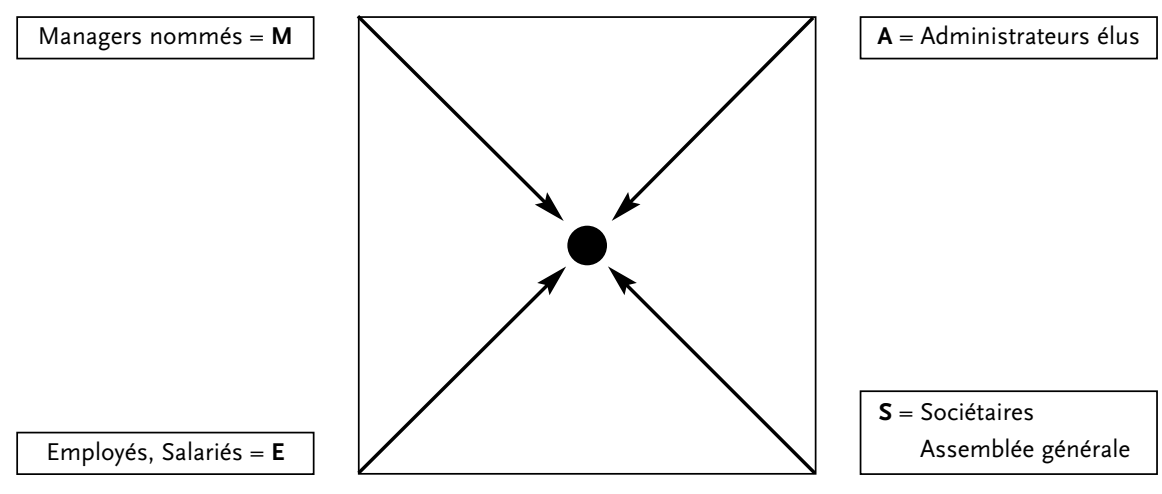

Dans les relations qui s'établissent entre les quatre pôles, deux clivages principaux sont susceptibles de se manifester: un clivage vertical, qui oppose les appareils de gestion aux réseaux d'associations, et un clivage horizontal, qui met à distance les leaders,

19. Le premier chapitre de l'ouvrage d'Henri Desroche sur le «projet coopératif» est consacré à «l'utopie coopérative» : «Le projet coopératif aura d'abord été un projet utopique. Aura été? Peut-être même fautil ajouter: est et sera à jamais un projet utopique. Expliquons-nous: bien sûr, identifier la coopération à une utopie, c'est pour l'acception commune la cataloguer comme irréalisable. [...] Mais [...] tenir à une telle objection c'est aussi s'en tenir à une réalité toute faite et même avouer implicitement qu'il n'y aura pas d'autre réalité que celle déjà immobilisée dans le fait accompli»(Desroche, 1976, p. 35). 
à la fois managers et administrateurs, de la population des sociétaires ou des employés ${ }^{20}$. Ce débat, qui traduit une tension permanente, est à nouveau évoqué dans la leçon inaugurale prononcée en novembre 2000 à l'École des HEC de Montréal par MarieClaire Malo. Après avoir présenté quelques auteurs classiques (Charles Gide, FrançoisAlbert Angers, Karl Polanyi...) et pris appui sur Henri Desroche, qui assura en collaboration avec Claude Vienney la direction de sa thèse (1980), M.-C. Malo insiste sur les rapports dialectiques entre identité et viabilité, projet rêvé et projet pratiqué, penseurs utopistes et disciples réalisateurs: «En économie sociale, la rencontre du groupement de personnes et de l'argent impose un compromis à l'un et à l'autre mais la combinaison est elle-même une innovation sociale. La combinaison existe non pas parce que nous sommes dans le meilleur des mondes mais pour faire naître le meilleur des mondes. On retrouve dans l'articulation entre un projet utopique et l'idéologie la même dynamique qu'à l'origine de la forme coopérative» (Malo, 2000, p. 29).

Le colloque interdisciplinaire que l'Université coopérative internationale (UCI), l'un des fleurons des réalisations d'Henri Desroche ${ }^{21}$, tient à l'Université Laval en 1980 entend faire le point sur le projet et les réalisations du système coopératif au Québec. Dans sa déclaration d'ouverture, François-Albert Angers, président du Centre interuniversitaire de recherche, d'information et d'enseignement sur les coopératives (CIRIEC, réseau québécois d'acteurs et de chercheurs engagés dans et sur les coopératives), présente le coopératisme comme «la troisième voie», parfaitement adaptée à la singularité québécoise: «Le Québec occupe peut-être une place privilégiée dans le monde, à ce sujet, parce qu'en tant que siège d'une nation qui a dû lutter pour sa survivance, les circonstances historiques l'ont mis devant la constatation que la formule coopérative constituait probablement, en matière de développement économique assuré sous le contrôle de sa population, la seule formule susceptible d'aboutir à des résultats vraiment efficaces» (Angers, 1981, p. 7). Il n’hésite pas à parler du développement coopératif au Québec comme d'une «tendance innée». Se référant à une conférence donnée par Henri Desroche à l'Université du Québec à Chicoutimi en maîtrise en gestion des petites et moyennes organisations, Paul Prévost estime de son côté que la philosophie de la coopération porte un projet de rénovation sociale qui dépasse largement l'implantation de la simple démocratie économique au sein de l'entreprise: les notions de créativité, de solidarité et de subsidiarité qui forment l'essence de la pensée coopérative pourraient constituer les pierres angulaires d'un modèle de comportement socioéconomique capable de guider les actions des coopératives et d'évaluer leur impact sur les collectivités régionales environnantes (Prévost, 1981, p. 197). Sans doute, parce qu'elle est soumise aux contraintes de systèmes dominants plus ou moins favorables, la coopération n'a-t-elle pas su dans le passé ériger un corpus conceptuel suffisam-

20. Dans ce travail d'élucidation des crises du système coopératif québécois, Henri Desroche se trouvera souvent associé à son collègue économiste Claude Vienney, qui interviendra, lui aussi, pour éclairer, entre autres, les relations entre administrateurs élus et professionnels de la gestion (Vienney, 1984).

21. Les activités de l'UCI et l'importance qu'y occupent chercheurs et acteurs québécois seront présentées plus avant. 
ment élaboré pour encadrer les actions des coopératives et des coopérateurs au-delà de la simple démocratie économique et organisationnelle, mais «ceux-ci sont aujourd'hui forcés d'assumer des responsabilités qui dépassent les cadres de la coopérative pour englober toute la société prise collectivement» (p. 202-203).

La conception de la sociologie que soutient Henri Desroche ${ }^{22}$ est de nature à inspirer une relecture des fondements et de l'évolution du système coopératif québécois. Les recherches, notamment doctorales, qu'il a dirigées ont en commun une visée interprétative largement commune, qui fait de la coopérative une forme institutionnelle ordonnée à protéger des intérêts socioéconomiques et idéologiques. Dans sa thèse consacrée à l'analyse de la formation des coopératives dans la seconde moitié du $\mathrm{XIX}^{\mathrm{e}}$ siècle, Benoît Tremblay met ainsi en exergue l'importance du facteur culturel: lorsque la perspective des promoteurs dépasse le niveau local d'organisation de certaines activités, «la formation et le développement des institutions coopératives sont très souvent présentés comme une manière privilégiée d'organiser les rapports socioéconomiques entre les Canadiens français et comme un moyen de conservation et d'affirmation de leur identité nationale» (Tremblay, 1982, p. 28). La coopérative est ainsi une façon pour les Canadiens français d'adopter une position de résistance parfaitement ajustée à leur situation de subordination à l'égard d'agents économiques dominants extérieurs à leur groupe. Benoît Lévesque, disciple d'Henri Desroche en sociologie des religions à l'Université de Sherbrooke puis son collaborateur en développement régional à l'Université du Québec à Rimouski, analyse pour sa part l'évolution du système coopératif québécois au cours du demi-siècle suivant comme l'une des modalités du passage d'un pareil projet de nationalisme de conservation à un projet de nationalisme économique. Au Québec, le développement de la coopération au cours de la première partie du Xx ${ }^{\mathrm{e}}$ siècle résulte en effet de la jonction de mouvements sociaux et d'institutions, à commencer par l'Église, dans le cadre d'un compromis social ordonné à la sauvegarde de l'ordre établi et au maintien des classes qui sont la base de la nation canadienne-française ${ }^{23}$ : «Pour l'élite clérico-nationaliste, [ce compromis] représentera un rempart contre le socialisme alors que pour les petits producteurs, fermiers et pêcheurs, il constituera un instrument susceptible de les préserver des effets d'un capitalisme les menaçant de disparition» (Lévesque, 1993, p. 77). Prises dans le mouvement de la Révolution tranquille, les entreprises coopératives adhéreront au processus de déconfessionnalisation et de modernisation de l'État; dans la mesure où elles font partie du capital francophone, elles profiteront en effet de la politique de nationalisme économique même s’il leur faudra mettre en sourdine leur anti-étatisme. Désormais,

22. Henri Desroche a assuré l'adaptation française de l'ouvrage d'Henrik Infield sur les entreprises communautaires (1955) ; l'ouvrage analyse en particulier des expériences coopératives d'inspiration religieuse dans des secteurs ruraux de la province de la Saskatchewan: la réalisation quaker de la Communauté de Penn-Craft ainsi que la Communauté inspirationniste d'Amana.

23. C'est aussi, dans une large mesure, la perspective que développe Claude Beauchamp, lui-même étroitement associé à l'action internationale d'Henri Desroche, dans son analyse du syndicalisme et de la coopération agricoles dans le Québec du premier tiers du XX⿳⺈冂大 siècle (Beauchamp, 1975). 
en effet, il s'agit moins de renforcer les institutions traditionnelles que sont la ferme familiale, la paroisse et la communauté villageoise que de contribuer au renforcement du contrôle québécois de l'économie. Dans la thèse qu'il consacre à l'action coopérative en Acadie, Joseph Yvon Thériault met l'accent, comme son condisciple Benoît Tremblay, sur la double fonction du mouvement coopératif: résistance à la transformation de l'économie et transition entre la société marchande et la société capitaliste: en limitant les effets négatifs propres à cette période de transition, le système coopératif «institutionnalise des mécanismes d'entrée dans la logique capitaliste pour des catégories marginalisées» (Thériault, 1981, p. 486).

Jointe à celle de l'économiste Claude Vienney, l'influence exercée par Henri Desroche et relayée par certains de ses disciples comptera indiscutablement dans le processus de reconnaissance de l'économie sociale au Québec. Cette reconnaissance était presque annoncée dans l'étude en forme à la fois de bilan et de prospective de B. Lévesque et M.-C. Malo (1992); elle devient officielle avec la création, en 1996, du Chantier de l'économie sociale. Parler de «reconfiguration» du modèle de Desroche en fonction de la spécificité québécoise dit alors à la fois la paternité de l'inspirateur (Desroche, 1983) et le travail d'ajustement et d'adaptation entrepris à sa suite sur fond de commune aspiration. Ce travail vise en particulier à affiner l'analyse des rapports entre les composantes «fondamentales» de l'économie sociale — les secteurs coopératif, mutualiste et associatif — et les composantes "périphériques» — les secteurs public, privé, syndical et communautaire. Le recours à la notion d'«interface» permet ainsi de «mieux cerner les frontières de l'économie sociale, de laisser de la place à la reconnaissance des mouvances et des migrations d'une zone à l'autre et finalement de tenir compte de phénomènes qui témoignent d'innovations économiques et sociales au sein des entreprises privées, du monde syndical et de l'État» (D’Amours et Malo, 1999, p. 18).

Dans les institutions universitaires où intervient Henri Desroche, des équipes de recherche spécialisées dans l'étude du mouvement coopératif profiteront de son appui intellectuel et de son réseau relationnel. C'est le cas de l'Institut de recherche et d'enseignement sur les coopératives de l'Université de Sherbrooke (IRECUS), né en 1974 de la transformation de la chaire de la coopération; du Centre de gestion des coopératives créé en 1975 à l'École des Hautes études commerciales de Montréal; enfin, du Centre canadien international de recherches et d'information sur l'économie publique et coopérative (CIRIEC), qui constitue lui-même un réseau de chercheurs. D’organisation à dominante anglophone lors de sa création en 1966, le CIRIÉC devient à compter de 1978 une association francophone centrée sur les coopératives; il s'ouvre à des collaborations internationales à travers, entre autres, l'Union coopérative internationale qu'anime Henri Desroche ${ }^{24}$.

24. En 1994, le champ d'études des membres du CIRIEC - devenu Centre interdisciplinaire de recherche et d'information sur les entreprises collectives — s'élargit à l'ensemble des entreprises d'économie sociale. Le titre de sa revue, Coopératives et développement, devient Économie et Solidarités. 
La dimension internationale des interventions d'Henri Desroche va prendre progressivement un nouvel élan. Il devient plus encore «animateur vagabond» et «colporteur de nouvelles». Ce faisant, $\mathrm{H}$. Desroche ouvre non seulement sur la France et l'Europe mais aussi et surtout sur les continents africain et sud-américain: la multiplicité de ses relations le conduit en effet à lancer en 1975 l'Université coopérative internationale (UCI), dite aussi l'Université des «quatre saisons », qui a contribué, pendant plus de dix ans, à consolider un réseau appuyé sur quatre pôles: le Québec, la France, l'Afrique noire et le Brésil25. À n'en pas douter, cette initiative a renforcé les relations entre les chercheurs québécois engagés sur le terrain des coopératives et du développement territorial et leurs collègues étrangers associés à l'UCI.

Henri Desroche considère l'UCI comme la troisième étape d'un processus qui a d'abord vu, à Paris, la création du Collège coopératif, puis, à l'échelle de la France, la formation d'un institut coopératif interuniversitaire (Desroche, 1980). Son inspiration va du projet de Charles Gide, qui crée en 1930 l'Institut international d'études coopératives, au discours que prononce le directeur de l'UNESCO à l'occasion du 500 anniversaire de l'Université d'Upsala, en septembre 1977: «Participer à [des] tâches collectives, apprendre des populations autant que par des études savantes devrait [...] constituer une des fonctions nouvelles de l'université dans les pays en développement» (M’Bow, 1978, p. 50). Dans l'organisation de cette université des «quatre saisons», nomade et «extra-muros», l'automne est généralement québécois. Celui de l'année 1978 voit ainsi l'UCI se déplacer successivement, à raison d'une semaine par site, de Montréal à Chicoutimi puis à Sherbrooke et enfin à Rimouski, où on comptera plus de 400 participants. Lors de cette dernière étape, organisée sur le thème Animation sociale, entreprises communautaires et coopératives (Lévesque, 1979), Henri Desroche livre sa conception de l'animateur, dont il trace quatre profils complémentaires: le maïeuticien, la cheville ouvrière, le négociateur et l'animateur vagabond (Desroche, 1979).

\section{Le développement des régions périphériques: autonomie et pro-activité}

La perspective que propose Henri Desroche dans le champ du développement régional se démarque des approches ambiantes, dominées par le paradigme de la dépendance (Favreau, 2000) ${ }^{26}$, dont on a perçu plus haut l'impact dans les interprétations qui sont alors données du développement des sciences sociales au Québec. On retrouve ici la marque d'une vision qui n'est pas sans rapport avec la tonalité utopiste et actionaliste de la pensée sociologique d'Henri Desroche; le développement des régions périphériques et l'avenir des petites communautés trouvent là des champs d'application sur lesquels a œuvré une génération de jeunes chercheurs en sciences sociales (sociologues,

25. Yves Hardy, «Un seul monde. Henri Desroche ou le souffle coopératif», Le Monde, 19 janvier 1987.

26. À propos du renouvellement de la notion de développement, Louis Favreau observe que ceux qui ont ouvert la voie sont les «économistes hétérodoxes des années 70-80», en marge du courant libéral comme du courant «dépendantiste»; au premier rang de ces innovateurs: Henri Desroche, Albert Meister, Paolo Freire et Yvan Illich, qui ont «remis en question l'économisme en s'interrogeant sur le rôle des associations et des coopératives dans le développement» (p. 7). 
géographes, psycho-sociologues, historiens...). Le développement social et économique des régions n'est pas pensé par rapport à un centre de commandement, c'est-à-dire en termes de subordination; il est avant tout conçu comme l'affaire de groupes territorialisés, dont il convient de conforter les compétences, de renforcer la mobilisation et de valoriser l'action collective (GRIDEQ, 1983; Dionne, 1979). Éventuellement à l'encontre de la vison étatiste et de la logique capitaliste, l'objectif est d'organiser la société — définie par son ancrage local — en communautés ${ }^{27}$. Dans sa thèse de doctorat, que dirige également Henri Desroche et qui traite de la mutation du secteur agricole dans l'Est du Québec, Bruno Jean se fait critique de la réduction du «développement » à une fonction linéaire de la croissance économique: la conjoncture théorique et sociopolitique permet, selon lui, de se demander s'il n'existerait pas des moyens d'améliorer la situation sociale autrement qu'en l'attendant de la seule sphère économique; il convie à profiter de la crise pour inventer de nouveaux modes de régulation sociale susceptibles d'anticiper une société solidaire (Jean, 1982, p. 482).

Dire que le développement ne se définit pas d'abord ni surtout par rapport à un centre étatique, c'est adopter un point de vue implicitement et partiellement critique à l'égard de l'«expérience» conduite au cours des années 1960 par le Bureau d'aménagement de l'Est du Québec (BAEQ), expérience qui s'inscrit dans ce que Jean-Jacques Simard qualifie d' «ère de gloire, avec ses hauts et ses bas, de la technocratie originale de la Révolution tranquille» (Simard, 1979, p. 65). L’arsenal déployé, qui se veut au service du développement des villes et des pôles de croissance, en est connu: enquêtes, participation, formation des leaders et animation. Ce dernier terme fait aussi partie du vocabulaire d'Henri Desroche, qui oppose toutefois à l'animation-intégration et à l'animation-contestation l'animation-médiation, qui a sa préférence en ce qu'elle permet, à ses yeux, d'opérer un ajustement réciproque et harmonieux des émissions-réceptions d'appareils avec les réceptions-émissions de réseaux. Les nombreux séjours d'Henri Desroche au Québec ont contribué à conforter et à tester ses connaissances théoriques et pratiques en la matière. Les premières relations qu'il y engage sont initiées à l'occasion du congrès de l'Association des sociologues de langue française qui s'est tenu en 1964 sous la présidence de Georges Gurvitch, à l'Université Laval, sur le thème: Les classes sociales dans le monde d'aujourd'hui. H. Desroche rend compte de cet événement et de ses prolongements dans la préface qu'il donne à l'ouvrage d'Aline Chèvrefils (1978) précisément consacré à l'animation et tiré d'une thèse qu'il a dirigée et qui porte en particulier sur le BAEQ:

27. Partant du concept de dépendance, Gilbert Renaud parvient à des conclusions similaires: «L'État national devient la négation et le principe même de la mort d'une communauté qui, sans lui, a toujours survécu à la domination en puisant ailleurs son structurant et en se dotant des mythes fondateurs nécessaires à sa vie et à sa régulation» (Renaud, 1984, p. 195). Dans la thèse qu'elle prépare sous la direction d'Henri Desroche et qui porte sur la contribution des petites et moyennes entreprises au développement régional, Anne-Marie Séguin estime que les PME contribuent tout au plus au «maintien» régional et qu'elles participent d'un processus de «dualisation à rebours» de l'économie (Séguin, 1982, p. 15-16). 
Je revenais de chantiers africains, et plus précisément sénégalais. Et notre surprise fut vive en apprenant que, dans notre communauté langagière, nous recourions de part et d'autre au même terme d'animation en lui conférant un contenu stratégique et tactique similaire. Même vogue et même vague. À l'autre bout de la chaîne, une bonne quinzaine d'années plus tard, j'aperçois l'hospitalière UQAR, à Rimouski, et notre ultime semaine d'université automnale dans le cadre de cette opération pilote pour une Université coopérative internationale en 1978 (dite UCI 78). [...] Enfin, entre ce point de départ et ce point d'arrivée, plusieurs autres opérations conjointes — ouvrages ou thèses — m'ont appris à fourrager dans plusieurs dossiers québécois: ceux du curé Labelle (G. Dussault), ceux de Louis Riel (G. Martel), ceux de telle ou telle congrégation religieuse (B. Lévesque), ceux du jeune Alphonse Desjardins en gésine de son utopie (B. Tremblay). Ces références latérales ne doivent pas être considérées comme insolites. À leur manière, chacune témoigne d'une animation in statu nascendi. (Desroche, 1978b, p. 4)

Le schème de pensée d'Henri Desroche qui prévaut dans l'analyse des coopératives trouve son équivalent fonctionnel dans celle du développement régional. Dans l'un et l'autre champs d'investigation et d'intervention, en effet, les mots d'ordre sont les mêmes: innovation, démocratie et développement. Ils sont au fondement de la création d'un certificat de deuxième cycle en développement des régions périphériques (1976) puis d'une maîtrise en développement régional (1978) au sein de l'Université du Québec à Rimouski (UQAR); à la rentrée universitaire de 2004, le programme de cette maîtrise souligne toujours que son objet, à visée interdisciplinaire, réside dans «les dimensions tant économiques que sociales, culturelles et géographiques du développement régional» et qu' «une attention particulière est accordée aux problèmes de développement des régions périphériques, tant dans les pays industrialisés que dans le Tiers-Monde».

Dans ce domaine du développement, la «boîte à outils» d'Henri Desroche contient un dispositif essentiel et original: la pratique de la recherche-action, qui est une autre forme d'effacement des frontières - entre enseignant et enseigné, cette fois - en ce qu'elle fonctionne à la façon d'une méthode inductive par valorisation des acquis individuels et collectifs. En septembre 1976, l'UQAR lance sa première Coopérative de recherche-action de l'Est du Québec (CRAEQ), sous la responsabilité de Benoît Lévesque, avec dix agents de développement, cinq professeurs ${ }^{28}$ et Henri Desroche comme animateur général des activités. Cette opération se fonde sur le caractère jugé prometteur de la combinaison de l'éducation et de la production, et du réinvestissement constant du savoir au service du développement territorial. Elle résulte aussi de la conviction acquise par les responsables universitaires que l'avenir de l'UQAR «était lié à celui de l'Est du Québec et que seul cet objet d'étude ne pouvait lui être enlevé par d'autres constituantes» (UQAR, 1976, p. 12). Cette même conviction avait déjà conduit à la création, en 1974, du Groupe de recherche interdisciplinaire sur le développement

28. En l'occurrence: Yves Dion, économiste, Clermont Dugas, géographe, Fernand Harvey et Benoît Lévesque, sociologues, et Guy Massicotte, historien. 
régional de l'Est du Québec (GRIDEQ), toujours en activité. L'UQAR se positionne dès lors explicitement comme l'un des partenaires actifs du développement de sa région.

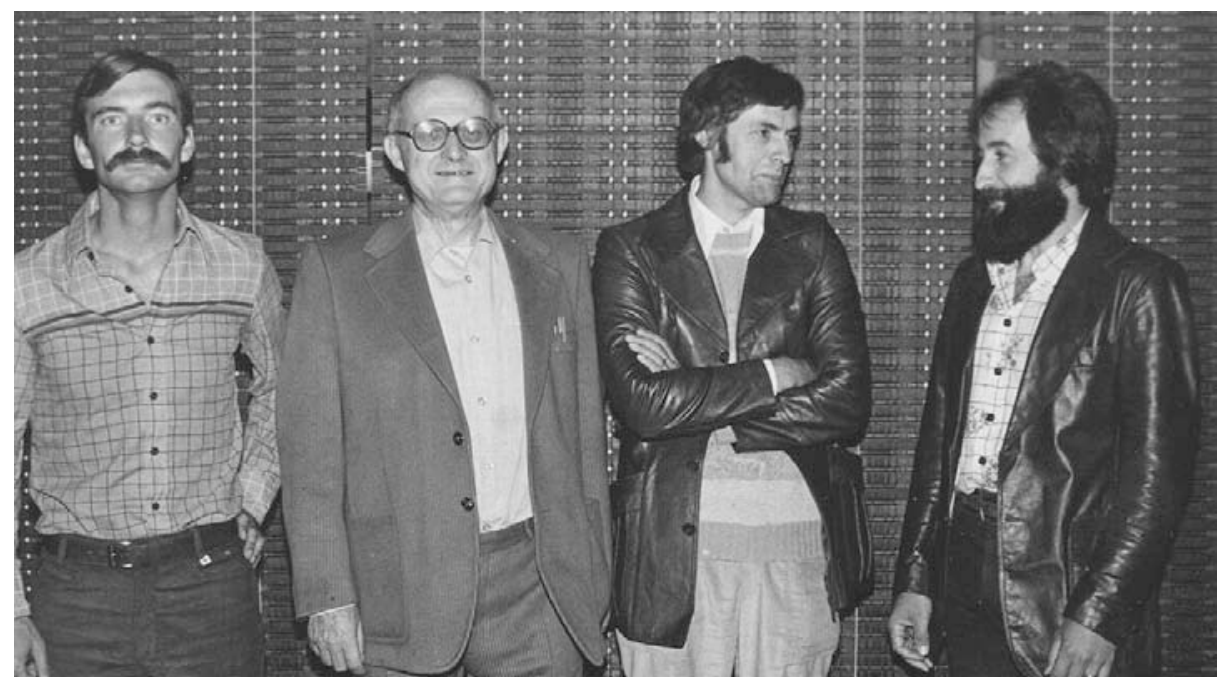

De gauche à droite: Paul Larocque (historien), Henri Desroche, Benoît Lévesque (sociologue, UQAM) et David Michaud (psychologue, décédé), 1978.

La CRAEQ s'inspire de deux ouvrages d'Henri Desroche: Le projet coopératif (1976) et Apprentissage en sciences sociales et éducation permanente (1971). Elle se veut

une association de type associatif formée d'agents de développement et de professeurschercheurs engagés dans une même entreprise de recherche-action à des titres divers. Pour les uns comme pour les autres, cette association suppose une certaine interversion des fonctions: les uns au plan pratique, les autres au plan intellectuel. Cet échange, qui place agents de développement et professeurs en état d'apprentissage, constitue une véritable surmultiplication des forces rendant plausible l'utopie d'une région capable de s'autoausculter selon les divers points de son territoire et selon ses dimensions socioculturelles et socioéconomiques. Cette utopie est liée de près à celle de l'autodétermination d'un développement régional. (UQAR, 1976, p. 7)

La formation, qui prend la forme d'un certificat en développement régional, comprend des rencontres collectives, des consultations individuelles ${ }^{29}$ et des visites sur le terrain.

29. Consultations dont le principe n'est pas étranger à la pratique de l' «autobiographie raisonnée» et où excellent les prédispositions maïeuticiennes d'Henri Desroche: «Comment communiquer en quelques minutes une expérience de travail? Par où commencer? Quelle dimension retenir? Qu'y a-t-il de véritablement intéressant dans cette action habituellement touffue? Plus d'un furent en mesure d'admirer l'art d'Henri Desroche: une ou deux questions précises de sa part suffisaient parfois pour que le stagiaire entrevoie comment son expérience pouvait être ventilée ou mise en perspective de deux ou trois façons différentes. Ainsi, un plan de recherches prenait graduellement forme et communiquait déjà à l'action entreprise de nouvelles perspectives» (UQAR, 1976, p. 19-21). 
«Desroche était un homme multiple, plein d'imagination et de cordialité, doté d'une capacité de travail assez exceptionnelle, aimant faire, construire, mettre sur pied, guider quelque temps... et puis laisser aller aux soins de ceux qu'il avait éveillés. Il repartait alors pour une entreprise nouvelle» (Séguy, 1994, p. 5). Le portrait que trace Jean Séguy traduit parfaitement la personnalité et l'activité multiple d'Henri Desroche, inventeur et éveilleur, éducateur et accoucheur, passé du statut de religieux à celui d'entrepreneur laïque. Ainsi se font et se défont ses réseaux, sur la double assise de l'utopie et du réalisme et au gré des projets en gestation.

Les réseaux québécois d'Henri Desroche sont singuliers. D’abord, la pluridisciplinarité de ses travaux a pu pleinement s'y déployer; même lorsque la sociologie de la coopération et celle du développement ont peu à peu évincé la sociologie de la religion, celle-ci est demeurée en filigrane, comme pour servir de matrice à l'analyse des valeurs et pour percer la raison ultime de l' «espérance pratiquée»: homme de vision et d'action, Henri Desroche reste «tenté par Les Dieux rêvés et demande pour eux droit de cité» (Isambert, 1997, p. 114). En second lieu, le Québec a offert à Henri Desroche toute une palette de projets et de réalisations qui s'ordonnaient parfaitement avec ses propres objets d'étude et sa prédilection pour les marges sociales et territoriales, à commencer par les «petites communautés» et la mobilisation des ressources locales du développement endogène; sa fécondité intellectuelle s'en est trouvée démultipliée. En outre, Henri Desroche intègre ses réseaux québécois dans des réseaux internationaux plus larges: il socialise pour ainsi dire ses collaborateurs à l'international, dans le cadre de l'UCI et de l'Institut de développement Nord-Sud, à La Pocatière, qui lui sert en ce domaine de bras séculier. Enfin, et surtout peut-être, son propre parcours individuel, religieux autant que professionnel, le porte à saisir les transformations passées et présentes de la société québécoise, que les jeunes chercheurs qu'il dirige inventorient et analysent avec quelques-unes des clefs interprétatives qu'il leur propose; l'hypothèse de l'homologie structurale paraît ainsi largement validée.

L'influence qu'Henri Desroche a pu exercer permet de mieux comprendre la spécificité et les dynamiques sociales d'un temps et d'un lieu. Pour l'un de ses anciens «compagnons», voulant signifier par là, d'une part, l'attrait que représentaient la séduction intellectuelle et la puissance de communication de son directeur de recherche, et, d'autre part, l'importance du changement social et culturel alors en cours et la recherche de nouveaux repères, «c'est le Québec qui s'est accroché à Desroche, et non l'inverse». Dès lors, la question du devenir de ces réseaux et de leur involution se pose moins en termes d'inévitable étiolement que de médiation et de postérité intellectuelle. L'un de ceux qui furent ses plus proches collaborateurs croit pouvoir dire, au lendemain de sa mort, en 1994, que «pour tous ceux et toutes celles qui, au Québec et au Canada français, mènent des recherches, enseignent ou interviennent dans le domaine de la coopération et l'économie sociale, Henri Desroche est non seulement une référence incontournable mais aussi une source d'inspiration toujours présente» (Lévesque, 1994-1995, p. 1). Tous les entretiens qui ont jalonné ce travail de sociologie de la connaissance sociologique ont fortement témoigné de cet attachement à un maître à 
penser... différemment. Au total, ils apprennent plus sur le Québec que sur Henri Desroche lui-même; ou plutôt, précisément, ils sont porteurs d'enseignements sur la signification et la portée de la forme réticulaire des relations qui se sont nouées entre l'un et l'autre.

\section{RÉSUMÉ}

L'influence exercée par Henri Desroche sur le processus d'internationalisation des sciences sociales au Québec, du milieu des années 1960 à celui des années 1980, a pris corps de part et d'autre de l'Atlantique. Au Québec même, d'abord, où $\mathrm{H}$. Desroche interviendra principalement à l'École des HEC de Montréal et dans les universités de Sherbrooke et de Rimouski. À Paris également, où de nombreux étudiants québécois vont suivre son enseignement et placer leur recherche sous sa direction au sein du Collège coopératif. L'analyse se fonde sur l'hypothèse selon laquelle la constitution de ses réseaux québécois tient à la relative homologie structurale entre l'individu Desroche, dans son propre parcours personnel et dans ses orientations intellectuelles et praxéologiques, et la culture politique d'un Québec de la fin ou d'après la Révolution tranquille en quête de nouveaux repères en matière d'action collective.

\section{ABSTRACT}

The influence of Henri Desroche on the process of internationalization of the social sciences in Quebec, from the mid-1960s to the mid-1980s, was felt on both sides of the Atlantic. In Quebec, first, where Desroche would teach mostly at Montreal's Ecole des hautes études commerciales and, in the regions, at the universities of Sherbrooke and Rimouski. In Paris, too, where many students from Quebec would follow his lectures and carry out their research projects under his direction within the Collège coopératif. This analysis is based on the hypothesis that the constitution of his Quebec networks depended on the relative structural equivalence between Desroche the individual, in his own personal career and in his intellectual and praxeological orientations, and the political culture of a Quebec, nearing the end or subsequent to the Quiet Revolution, in search of new points of reference as regards collective action.

\section{RESUMEN}

La influencia ejercida por Henri Desroche sobre el proceso de internacionalización de las ciencias sociales en Quebec, de mitad de los años sesenta a los años ochenta, tomó cuerpo de una a otra parte del Atlántico. En Quebec, en primer lugar, dónde H. Desroche intervendrá principalmente en la Escuela de HEC de Montreal y, en región, en las Universidades de Sherbrooke y de Rimouski. En París también, dónde numerosos estudiantes de Quebec van a seguir su enseñanza y a colocar la investigación bajo su dirección en el Colegiado cooperativo. El análisis se basa en la hipótesis según la cual la constitución de sus redes en Quebec tiene que ver con la relativa homología estructural entre el individuo Desroche, en su propio itinerario personal y en sus orientaciones intelectuales y praxéologicas, y la cultura política de un Quebec del final o posterior a la Revolución tranquila en búsqueda de nuevos puntos de referencia en cuanto a la acción colectiva. 


\section{BIBLIOGRAPHIE}

Angers, F.-A. (1981), «Déclaration d'ouverture», in C. BEAUChamp (dir.), Le développement des coopératives au Québec d'ici l'an 2000, Actes du colloque de l'Université coopérative internationale tenu à l'Université Laval du 24 au 27 août 1980, p. 1-12.

BeAuchamp, C. (1975), Coopération et syndicalisme agricoles au Québec (1900-1930), Paris, ÉPHÉ, thèse de doctorat de $3^{\mathrm{e}}$ cycle en sociologie.

Chèvrefils, A. (1978), Le rôle des animateurs sociaux, Montréal, Éditions coopératives Albert Saint-Martin (préface d'Henri Desroche).

Chèvrefils, A. (1968), Le rôle et la formation des animateurs sociaux, à partir de trois expériences d'animation au Canada français, Université de Paris, École pratique des hautes études (VIe section), thèse de doctorat de $3^{\text {e }}$ cycle (sous la dir. d'Henri Desroche).

Claudel, P. (1965), «Parabole d'Animus et d'Anima: pour faire comprendre certaines poésies de Rimbaud», in Positions et propositions (Bibl. de la Pléiade), Paris, NRF.

D’Amours, M. et M.-C. Malo (1999), Modèle québécois d'économie sociale: reconfiguration du modèle de Desroche, Cahiers du LAREPPS, Cahiers du CRISES, $\mathrm{n}^{\circ}$ 99-04.

Deconchy, J.-P. et G. Martel (1974), Idéologie chrétienne de l'interaction sociale et lecture de résultats d'investigation, Paris, Laboratoire de psychologie sociale, Sherbrooke, Faculté de théologie.

Degenne, A. et M. Forsé (1994), Les réseaux sociaux. Une analyse structurale en sociologie, Paris, Armand Colin.

Denault, B. et B. LÉvesque (1975), Eléments pour une sociologie des communautés religieuses au Québec, Sherbrooke, Université de Sherbrooke, Montréal, Les Presses de l’Université de Montréal.

Desroche, H. (1990), «Des républiques d'enfants à des universités en compagnonnage», Anamnèses, 1990, $\mathrm{n}^{\circ} 4$.

Desroche, H. (1984), Itinérante et saisonnière: une université coopérative internationale (1977-1984), Université coopérative internationale.

Desroche, H. (1983), Pour un traité d'économie sociale, Paris, Coopérative d'information et d'édition mutualiste.

Desroche, H. (1980), «Itinérances d'une collégialité. D’un Collège Coopératif parisien à une Université Coopérative internationale ", Archives des sciences sociales de la coopération et du développement, $\mathrm{n}^{\circ} 52$ 53, avril-sept., p. 1-5.

Desroche, H. (1979), «Animation et éducation permanente», in B. LÉVESQUE (dir.), Animation sociale, entreprises communautaires et coopératives, Montréal, Éd. coopératives Albert Saint-Martin, p. 201 211.

Desroche, H. (1978a), Éducation permanente et créativités solidaires. Lettres ouvertes sur une utopie d'université hors les murs (Apprentissage 2), Paris, Éd. Ouvrières.

Desroche, H. (1978b), «Préface», in ChÈvrefils, Aline, 1978, Le rôle des animateurs sociaux, Montréal, Éditions coopératives Albert Saint-Martin.

Desroche, H. (1976), Le projet coopératif. Son utopie et sa pratique. Ses appareils et ses réseaux. Ses espérances et ses déconvenues, Paris, Éd. Économie et Humanisme, Éd. Ouvrières.

Desroche, H. (1973), Sociologie de l'espérance, Paris, Calmann-Lévy.

Desroche, H. (1972), L’homme et ses religions. Sciences humaines et expériences religieuses, Paris, Éd. du Cerf.

Desroche, H. (1971), Apprentissage en sciences sociales et éducation permanente, Paris, Éd. Ouvrières.

Desroche, H. (1969), Le développement intercoopératif: ses modèles et ses combinaisons, Sherbrooke, Librairie de la Cité universitaire, Les Cahiers de la coopération $\left(\mathrm{n}^{\circ} 1\right)$.

Desroche, H. (1955), Les Shakers américains. D’un néo-christianisme à un pré-socialisme?, Paris, Éd. de Minuit.

Desroche, H. et A. Meister (1955), Une communauté de travail de la banlieue parisienne, Paris, Éd. de Minuit. 
Desroches, H.-C. (1944), «Dialectique de la “communauté” », in Desroches, H.-C. et alii, Caractères de la communauté, Écully, Économie et humanisme.

Dion, M. et L. Mélançon (1996), «Lucien Vachon: un théologien dans la cité», in M. Dion et L. MéLANÇON (dir.), Un théologien dans la cité. Hommage à Lucien Vachon, Montréal, Éd. Bellarmin, coll. «Recherches».

Dionne, H. (1979), «Animation et recherche en vue d'un développement régional», in B. LÉVESQUE (dir.) (1979), Animation sociale, entreprises communautaires et coopératives, Montréal, Éd. coopératives Albert Saint-Martin, p. 212-222.

Dofny, J. (1978), «Les stratifications de la société québécoise», Sociologie et sociétés, vol. 10, nº 2, p. 87-102.

Dumont, F. (1997), Récit d’une émigration. Mémoires, Montréal, Boréal.

Dussault, G. (1975), Messianisme, utopie et colonisation au Québec (1850-1900). Étude sociohistorique d'un cas: le curé Labelle, Paris, ÉPHÉ (VI ${ }^{\mathrm{e}}$ section), Université Paris V, Thèse de doctorat en sociologie (sous la dir. d'Henri Desroche).

Dussault, G. et G. Martel (1976), Communautés religieuses et économie. Rapport de recherche 1975-76. Les 5 premières communautés masculines établies au Québec sous le régime anglais (1837-1870), Université de Sherbrooke, Faculté de théologie.

FABRe, G. (2004), «Un arc transatlantique et sa tangente ou comment se dessine un réseau intellectuel franco-québécois?», Globe. Revue internationale d'études québécoises, vol. 7, nº 1, p. 43-78.

Favreau, L. (2000), Économie sociale, coopération internationale et développement des sociétés du Sud, Cahiers du CRISES, $\mathrm{n}^{\circ} 18$.

Fournier, M. (1973), Institutionnalisation et différenciation de disciplines dans une situation de double dépendance, Paris, École pratique des hautes études, thèse de doctorat de $3^{\mathrm{e}}$ cycle.

Fournier, M. (1972), «De l'influence de la sociologie française au Québec», Revue française de sociologie, XIII, suppl., p. 630-665.

Fournier, M. et Maheu, L. (1975), «Nationalismes et nationalisation du champ scientifique québécois», Sociologie et sociétés, vol. 7, n 2, p. 89-114.

Fournier, M., A. Germain, Y. Lamarche et L. Maheu (1975), «Le champ scientifique québécois: structure, fonctionnement et fonctions», Sociologie et sociétés, vol. $7, \mathrm{n}^{\circ} 1$.

GAGNON, G. (1978), «Sociologie, mouvements sociaux, conduites de rupture: le cas québécois», Sociologie et sociétés, vol. $10, \mathrm{n}^{\circ} 2$, p. 103-121.

GRIDEQ, (1983), Aménagement intégré des ressources et luttes en milieu rural, UQAR.

GRIDEQ et Institut DE DÉvelopPEMENT NoRd-Sud, 1989, Les villages ruraux menacés: le pari du développement, Université du Québec à Rimouski, GRIDEQ.

INFIELD, H. (1955), Coopératives communautaires et sociologie expérimentale. Esquisses pour une sociologie de la coopération, Paris, Éd. de Minuit (traduit de Utopia and Experiment).

Isambert, F.-A. (1997), «Une sociologie de l'espérance», in É. Poulat et C. Ravelet (dir.), Henri Desroche, un passeur de frontières, Paris, L'Harmattan, p. 93-114.

JEAN, B. (1982), L'agriculture périphérique dans l'Est du Québec, Paris, EHESS, thèse de doctorat en sociologie (sous la dir. d'Henri Desroche).

Lemieux, V. (1999), Les réseaux d'acteurs sociaux, Paris, PUF, coll. «Sociologies».

LÉvesque, B. (1994-1995), «En hommage à Henri Desroche, sociologue des religions et de la coopération (1914-1994)», Coopératives et développement, vol. 26, nº 1, p. 1-7.

LÉvesque, B. (1993), «Les coopératives au Québec: deux projets distincts pour une société?, in N. BÀRdOSFÉLTORONY et alii, Coopération: défis pour une démocratie économique, Bruxelles, Éd. Vie ouvrière, Actes de la Chaire interuniversitaire de coopération, p. 69-95.

LÉvesque, B. (dir.) (1979), Animation sociale, entreprises communautaires et coopératives, Montréal, Éd. coopératives Albert Saint-Martin.

LÉvesque, B. (1975), D’un projet primitivement utopique à une congrégation religieuse. Sociologie génétique des clercs de Saint-Viateur (1793-1859), ÉPHÉ (VI ection), Université de Paris V, Thèse de doctorat en sociologie (sous la dir. d'Henri Desroche avec la collaboration de Jean Séguy). 
LÉVesque, B. et M.-C. Malo (1992), «L'économie sociale au Québec: une notion méconnue, une réalité économique importante», in J. Defourny et J. L. Monzon CAmpos (dir.), Économie sociale, entre économie capitaliste et économie publique/The Third Sector, Cooperative, Mutual and Nonprofit Organizations, CIRIEC, De Boeck Université, Bruxelles.

Maheu, L. (1980), «Pouvoir et société au Québec: le problème de l'État et les appareils d'État», in ACSALF, La transformation du pouvoir au Québec, Colloque 1979, Montréal, Éd. coopératives Albert SaintMartin.

Maheu, L. (1974), Enseignement supérieur et structure sociale: les fonctions sociales de l'Université québécoise, Paris, École pratique des hautes études, Thèse de doctorat de $3^{\mathrm{e}}$ cycle.

Malo, M.-C. (2000), La coopération et l'économie sociale, Montréal, École des hautes études commerciales (Les Cahiers des leçons inaugurales).

Malo, M.-C. (1980), Émergence, fonctionnement et transformation de l'organisation coopérative des consommateurs québécois (1938-1978), Paris, ÉHÉSS, Thèse de $3^{\mathrm{e}}$ cycle en sciences sociales du développement (sous la dir. d'Henri Desroche).

Malo, M.-C. et M. Bouchard (2001), «En hommage à Claude Vienney (1929-2001), Économie et Solidarités, $32, \mathrm{n}^{\circ} 1-2$, p. 1-12.

Martel, G. (1976), Le messianisme de Louis Riel: 1844-1885, Université Paris 10, EHESS, thèse de troisième cycle de sociologie (sous la direction d'Henri Desroche).

M'Bow, A. M. (1978), «Le rôle des universités dans les pays en voie de développement», Archives des sciences sociales de la coopération et du développement, 43, 1978.

Poulat, É. (1997), «Henri Desroche, compagnon et maître», in É. Poulat et C. Ravelet (dir.), Henri Desroche, un passeur de frontières, Paris, L'Harmattan, p. 9-13.

Prévost, P. (1981), «La coopération et le développement régional», in C. BEAUCHAMP (dir.), Le développement des coopératives au Québec d'ici l'an 2000, Actes du colloque de l'Université coopérative internationale tenu à l'Université Laval du 24 au 27 août 1980, p. 195-203.

Renaud, G. (1984), À l'ombre du rationalisme. La société québécoise, de sa dépendance à sa quotidienneté, Montréal, Éd. coopératives Albert Saint-Martin.

Ryan, N. (1969), «Situation actuelle des sciences religieuses au Canada français», Archives de sociologie des religions, 27, p. 97-118.

SÉGUIN, M.-T. (1982), Petites et moyennes entreprises et développement régional: l'heure de la restructuration de l'appareil de production: une enquête sur cette question dans une région québécoise: le Saguenay-LacSaint-Jean, ÉHÉSS, Thèse de $3^{e}$ cycle de sociologie (sous la dir. d'Henri Desroche).

SÉGUY, J. (1994), «In memoriam. Henri Desroche (1914-1994)», Archives de sciences sociales des religions, 87, p. 5-12.

SÉGUY, J. (1971), «Une sociologie des sociétés imaginées: monachisme et utopie», Annales, mars-avril, p. 328-354.

Simard, J.-J. (1979), La longue marche des technocrates, Montréal, Ed. coopératives Albert Saint-Martin.

Thériault, J. Y. (1981), Acadie coopérative et développement acadien: contribution à une sociologie d'un développement périphérique et à ses résistances, Paris, EHESS, thèse de doctorat de sociologie (sous la dir. d'Henri Desroche).

Touraine, A. (1997), «Henri Desroche et la communauté exemplaire», in E. Poulat et C. Ravelet (dir.), Henri Desroche, un passeur de frontières, Paris, L'Harmattan, p. 17-22.

Touraine, A. (1978), «Théorie et pratique d'une sociologie de l'action », Sociologie et Sociétés, vol. 10, n 2, p. 149-188.

Tremblay, B. (1982), Analyse socioéconomique de la formation des organisations coopératives: le cas du Québec (1850-1914), Paris, EHESS, thèse de doctorat de troisième cycle (sous la dir. d'Henri Desroche, avec la collaboration de Claude Vienney). 
UNIVERSITÉ DU QUÉBEC À RIMOUSKI, (1976), L'opération CRAEQ (Coopérative de recherche-action de l'Est du Québec), UQÀR.

Vienney, C. (1994), L'économie sociale, Paris, Éd. La Découverte, coll. « Repères».

VIENNEY, C. (1984), «La formation des administrateurs de coopératives. Observations et inductions à partir d'études comparées France-Québec ", Archives de sociologie de la coopération et du développement, p. 109-122. 